\title{
HEMATOMA EPIDURAL LOMBAR PÓS-CIRURGICO EM PACIENTE COM LEUCEMIA. RELATO DE CASO
}

\author{
POSTOPERATIVE LUMBAR EPIDURAL HEMATOMA IN A PATIENT \\ WITH LEUKEMIA. CASE REPORT
}

\section{HEMATOMA EPIDURAL LUMBAR POSQUIRÚRGICO EN PACIENTE CON LEUCEMIA. RELATO DE CASO}

Wagner Pasqualin ${ }^{1}$, Marcos Antonio Tebet' ${ }^{2}$, Mareio Oliveira Penna de Carvalho ${ }^{3}$

\begin{abstract}
RESUMO
A ocorrência de hematoma epidural como complicação pós-cirúrgica é relativamente baixa. O reconhecimento dessa patologia no diagnóstico diferencial nas paraplegias pós-cirúrgicas imediatas e o tratamento precoce por meio de intervenção cirúrgica com a descompressão do canal são fatores que estão diretamente relacionados à melhora do quadro neurológico. Este relato de caso é de um hematoma epidural no pós-operatório imediato de descompressão por estenose do canal vertebral lombar em paciente com leucemia.
\end{abstract}

Descritores: Hematoma epidural espinal; Paraplegia/complicações; Procedimentos cirúrgicos operatórios.

\begin{abstract}
The occurrence of epidural hematoma as a postoperative complication is relatively low. The recognition of this condition in the differential diagnosis in the immediate postoperative paraplegia and the early surgical decompression are directly related with neurological improvement. We report a case of epidural hematoma in the early postoperative period of surgical decompression of the lumbar spinal canal, in a patient with leukemia.
\end{abstract}

Keywords: Hematoma, Epidural, Spinal; Paraplegia/complications; Surgical procedures, operative.

\section{RESUMEN}

La ocurrencia de hematoma epidural, como complicación posquirúrgica, es relativamente baja. El reconocimiento de esa patología, en el diagnóstico diferencial en las paraplejías posquirúrgicas inmediatas y el tratamiento precoz por medio de intervención quirúrgica con la descompresión del canal, son factores que se relacionan directamente con la mejoría del cuadro neurológico. Este relato de caso es de un hematoma epidural en el posoperatorio inmediato, después de descompresión, por estenosis, del canal vertebral lumbar en paciente con leucemia.

Descriptores: Hematoma epidural espinal; Paraplejía/complicaciones; Procedimientos quirúrgicos operatorios.

INTRODUÇÃO

O hematoma epidural é uma complicação incomum no pós-operatório da cirurgia da coluna vertebrall, ${ }^{1,2}$. Jackson ${ }^{3}$, em 1869 foi o primeiro a descrever esta patologia em crianças e a definiu como uma urgência cirúrgica.

A maioria dos pacientes submetidos a procedimento cirúrgico na coluna apresentará hematoma epidural pequeno e clinicamente insignificante ${ }^{3,4}$. Entretanto, a prevalencia de hematoma epidural pós-cirúrgico com déficit neurológico e significado clínico é muito pequena, variando de 0,1 a 0,22\%,6. Em ordem decrescente de frequencia hematomas epidurais pós-cirúrgicos ocorrem nos segmentos torácico, cervical e lombar².

Várias etiologias relacionadas com o desenvolvimento de hematomas epidurais têm sido relatadas, inclusive trauma, coagulopatias de diversas causas, condições reumáticas, pós-operatória e por neoplasias. São denominados "hematomas epidurais espontâneos" aqueles sem etiologia aparente ou fator de risco associado ${ }^{2,7,8}$.

Os fatores pré-operatórios que influenciam com aumento do risco de hematoma epidural pós-cirúrgico são o uso de anticoagulantes ou anti-inflamatórios, paciente idoso, índice de massa corporal (IMC) elevado, abordagem cirurgica de múltiplos níveis da coluna vertebral, cirurgias prévias e coagulopatias 2,5,9. Existem também fatores pós-operatórios como a drenagem insuficiente.

Dentre os fatores de risco, a presença de coagulopatia pré-operatória é, segundo Kou et al. ${ }^{6}$ um dos fatores mais importantes. Uma das causas de coagulopatia que com frequencia deixa de ser diagnosticada pré-operatoriamente é a leucemia.

Neste trabalho é apresentado o relato clínico de um paciente do sexo feminino com 67 anos de idade, com hematoma epidural pós-cirúrgico que evoluiu com déficit neurológico grave.

\section{RELATO DO CASO}

Paciente do sexo feminino, 67 anos de idade, apresentava claudicação neurológica intermitente com piora progressiva há um ano. Durante este período foi submetida a tratamento clínico sem melhora da sintomatologia. Apresentava exame físico normal, com teste de Lasègue negativo e sem alterações sensitivas ou motoras objetivas, com pulsos distais em membros inferiores normais, foi afastada insuficiência vascular em membros inferiores em exame de eco doppler.

O exame radiológico da coluna lombar mostrava alterações degenerativas com osteófitos e diminuição da altura do disco em L2-L3 e L3-L4. As imagens por ressonância magnética (RM) mostraram

1. Mestre, Professor Colaborador na Disciplina de Ortopedia eTraumatologia da Faculdade Ciências Médicas da Universidade de Campinas - Unicamp - Campinas (SP), Brasil
2. Doutor, Professor Adjunto da Disciplina de Ortopedia eTraumatologia da Faculdade de Medicina de Jundiaí - Jundiaí, SP, Brasil.

3. Mestre em Ciências Médicas pela Faculdade de Medicina da Universidade de São Paulo (HCFM-USP)

Correspondência: Instituto da Coluna. Rua: Abílio Figueredo, 92 - Ed. Nino Plaza - Jundiaí - SP.Brasil. Cep.: 13.206-140. Email.: institutodacoluna@institutodacoluna.com.br. 
estenose do canal vertebral em L2-L3 e L3-L4 central e estenose foraminal (Figura 1) além de gânglios abdominais que nao foram inicialmente valorizados.

Com o diagnóstico de estenose do canal vertebral lombar estabelecido e a falência do tratamento clínico foi proposta a cirurgia. Como rotina, foram solicitados exames pré-operatórios, que se mostraram normais, sem alterações na contagens de células brancas, vermelhas, plaquetas e sem sinais de discrasias sanguíneas ou qualquer outro sinal de coagulopatia. A avaliação de risco cirúrgico pré-anestésico foi ASA II, e como antecedente pessoal a paciente apresentava apenas hipertensão arterial sistémica controlada.

Trinta dias após avaliação pré-operatória foi submetida a tratamento cirúrgico, sendo realizada a descompressão do canal com laminectomia total de L3, foraminotomia e artrodese dos segmentos de L2-L3 e L3-L4, estes segmentos foram estabilizados com parafusos pediculares. Não houve qualquer complicação intraoperatória, o tempo cirúrgico aproximado foi de três horas com perda sanguínea de 400 $\mathrm{ml}$, não havendo a necessidade de transfusão de sangue ou hemoderivados. Foi utilizado, como rotina dreno de sucção por 48 horas.

Após recuperação anestésica o exame físico não apresentava qualquer alteração neurológica ASIA V, tendo sido encaminhada a UTI para cuidados pós-operatório imediatos. Exames laboratoriais de controle no primeiro dia de pós-operatório, demonstraram leucocitose de $143.000 \mathrm{~mm}^{3}$, tendo sido feito a hipótese diagnostica de uma leucose. O dreno de sucção apresentou débito de $200 \mathrm{ml}$ nas primeiras 12 horas. Com 14 horas de pós-operatório a paciente recebeu alta da Unidade de Terapia Intensiva, sem apresentar qualquer alteração neurológica (ASIA V). Com 24 horas de cirurgia o exame neurológico demonstrava paraparesia de membros inferiores simétrica (ASIA II) sem alteração em reflexos tendinosos profundos, sem alterações esfincterianas. Foi realizada ressonância magnética lombar cerca de 6 horas após o inicio do quadro neurológico, momento no qual a paciente apresentava quadro de paraplegia, com alteração esfincteriana vesical (ASIA I).

A RM demonstrou hematoma epidural com compressão do saco dural nos níveis de T12 a L3 (Figura 2). Realizou-se a drenagem cirúrgica do hematoma aproximadamente 12 horas após o inicio do quadro neurológico (Figura 3).

No pós-operatório imediato a paciente já demonstrou alguma recuperação de déficit neurológico e com cinco meses apresentava exame neurológico normal ASIA V, com recuperação completa, sem qualquer sequela.

No segundo dia após a primeira cirurgia foi solicitada avaliação do hematologista que solicitou mielograma e confirmou o diagnóstico de Leucemia Linfocítica.

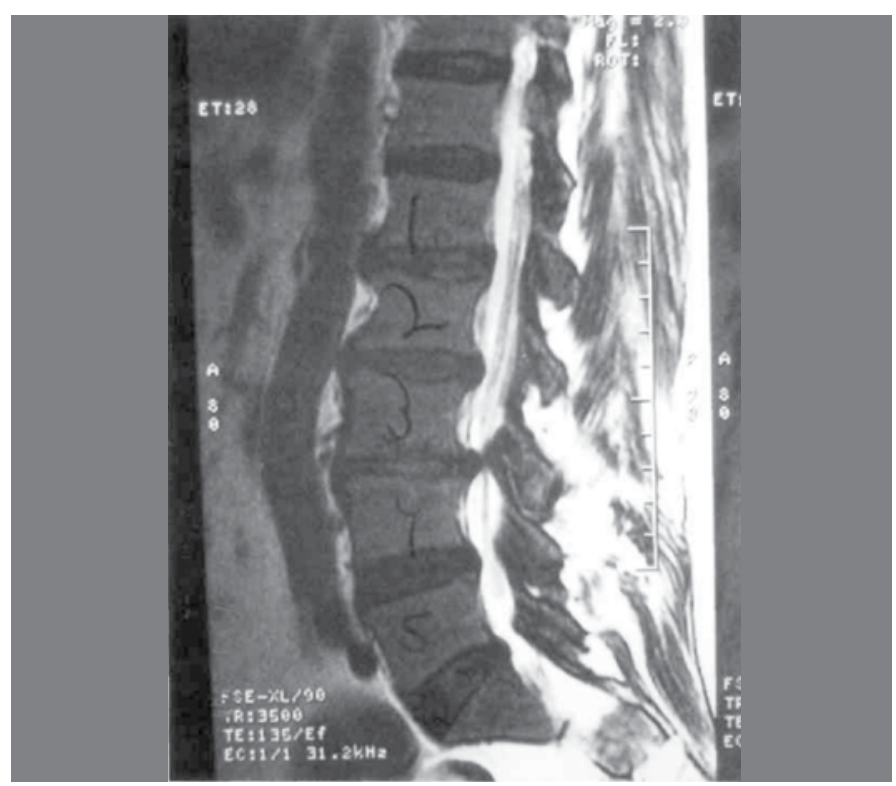

Figura 1. Imagem de ressonância magnética com estenose do canal lombar.

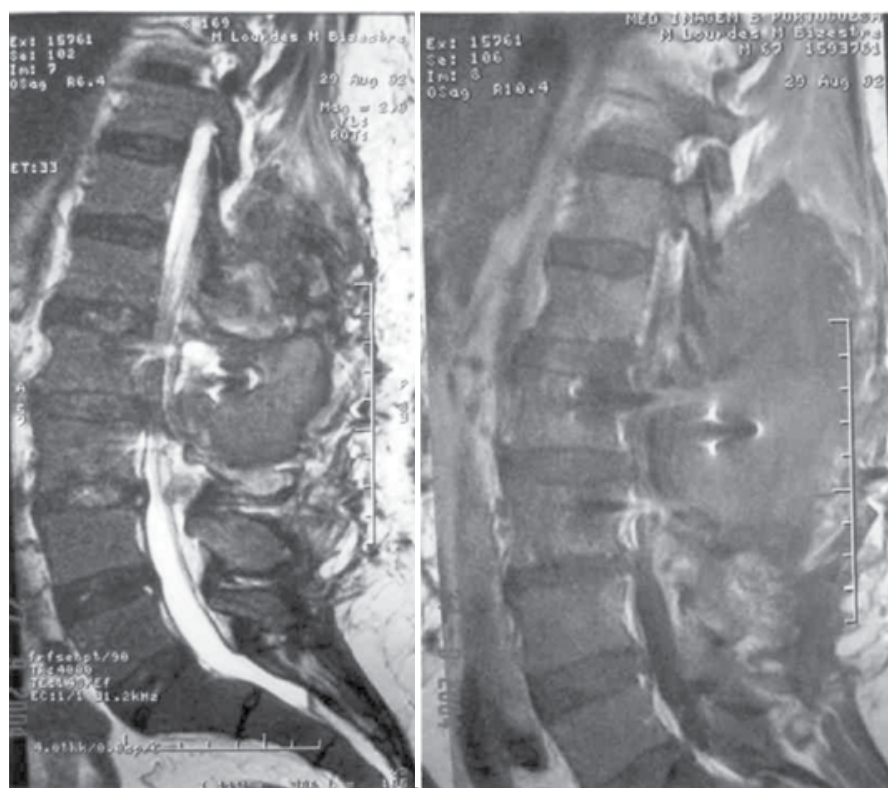

Figura 2. Ressonância magnética mostrou extenso hematoma epidural de T12 a L3.

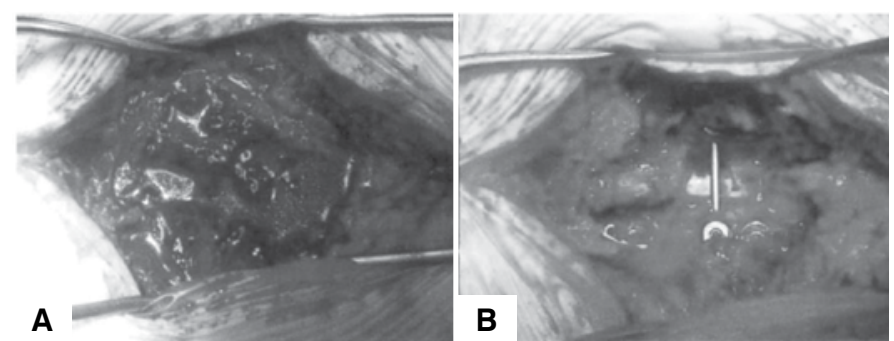

Figura 3. (A) Imagem intra-operatória do hematoma epidural; (B) Após drenagem cirúrgica.

\section{DISCUSSÃO}

O hematoma epidural pós-cirúrgico é uma complicação grave que pode levar a déficit neurológico. As causas desta complicação não estão totalmente esclarecidas. Groen e Ponssen ${ }^{10}$ concluíram em uma série de estudos que a origem da hemorragia na formação do hematoma epidural é a ruptura do plexo venoso vertebral interno e quanto maior a exposição cirúrgica maior a probabilidade de lesionar esses vasos.

O quadro clínico normalmente encontrado no pós-operatório é dor aguda de forte intensidade no local da cirurgia, evoluindo com diminuição da força motora progressiva,

parestesia e alterações sensitivas, podendo cursar com alterações esfincterianas e síndrome da cauda equina, como observado em nosso caso $^{7,12}$.

No caso apresentado, o déficit neurológico iniciou-se 12 horas após a recuperação anestésica, onde o paciente nesse período permaneceu sem alteração neurológica. A razão para o atraso no desenvolvimento das alterações neurológicas não são definidas na literatura ${ }^{2,13}$

O exame de eleição para o diagnóstico do hematoma epidural pós-cirúrgico é a Ressonância Magnética ${ }^{2,7}$. O diagnóstico diferencial com perda neurológica em pós-operatório deve ser feito com hemorragia intradural, compressão medular por estruturas extra-axiais, infecção, condições inflamatórias e infarto medular

Os fatores prognósticos para a recuperação neurológica ficam estabelecidos entre a força de compressão e o tempo de duração da compressão. Quanto ao tempo, a recuperação completa do déficit neurológico pode ser obtida se a descompressão for realizada até dez horas após o início das alterações neurológicas ${ }^{13-15}$. 
Vandermeulen et al. ${ }^{16}$ observaram que a maioria dos seus pacientes com hematoma epidural espontâneo, que foram submetidos à descompressão cirúrgica dentro de 8 horas, obteve recuperação neurológica total ou parcial.

Kou et al. ${ }^{6}$ realizaram estudo para identificar os fatores de risco relacionados à ocorrência de hematoma epidural pós-cirúrgico, concluíram que procedimentos cirúrgicos de múltiplos níveis e a presença de coagulopatia pré-operatória foram os únicos fatores significativos. Salientam que a presença de coagulopatia nem sempre é relatada pelo paciente ou não é diagnosticada no pré-operatório.

A descompressão associada ou não a artrodese posterior da coluna está indicada no tratamento da estenose do canal lombar quando houver falência do tratamento clínico. Cabana et al. ${ }^{17} \mathrm{em}$ uma revisão de 1.487 cirurgias da coluna tiveram 15 casos de hematoma epidural, sete na coluna lombar sendo a estenose do canal a indicação para a primeira cirurgia em seis destes casos.
Apesar de alguns autores sugerirem o tratamento conservador em casos em que é conhecida a presença de coagulopatia com alterações neurológicas leves ${ }^{8}$ e da pouca experiência na literatura, o tratamento recomendado pela maioria dos autores é o tratamento cirúrgico com laminectomia e descompressão, que deve ser realizada o mais rápido possível para minimizar as sequelas neurológicas ${ }^{7,8}$.

\section{CONSIDERAÇÕES FINAIS}

O hematoma epidural é uma patologia que deve ser lembrada na presença de déficit neurológico após cirurgia da coluna vertebral.

A possibilidade de coagulopatia deve ser investigada em todos os pacientes que serão submetidos a tratamento cirúrgico da coluna vertebral.

Na presença de hematoma compressivo e déficit neurológico a descompressão deve ser procedimento realizado de emergência.

\section{REFERÊNCIAS}

1. Mayfield FH. Complications of laminectomy. Clin Neurosurg. 1976:23:435-9.

2. Uribe J, Moza K, Jimenez O, Green B, Levi AD. Delayed postoperative spinal epidural hematomas. Spine J. 2003;3(2):125-9.

3. Jackson R. Case of spinal apoplexy. Lancet. 1869:2:5-6.

4. Kotilainen E, Alanen A, Erkintalo M, Helenius H, Valtonen S. Postoperative hematomas after successful lumbar microdiscectomy or percutaneous nucleotomy: a magnetic resonance imaging study. Surg Neurol. 1994;41(2):98-105.

5. Teplick JG, Haskin ME. Review. Computed tomography of the postoperative lumbar spine. AJR Am J Roentgenol. 1983;141(5):865-84.

6. Kou J, Fischgrund J, Biddinger A, Herkowitz H. Risk factors for spinal epidural hematoma after spinal surgery. Spine (Phila Pa 1976). 2002;27(15):1670-3

7. Scavarda D, Peruzzi P, Bazin A, Scherpereel B, Gomis P, Graftieaux JP, et al. [Postoperative spinal extradural hematomas. 14 cases]. Neurochirurgie. 1997;43(4):220-7.

8. Pecha MD, Able AC, Barber DB, Willingham AC. Outcome after spontaneous spinal epidural hematoma in children: case report and review of the literature. Arch Phys Med Rehabil. 1998;79(4):460-3.

9. Groen RJ. Non-operative treatment of spontaneous spinal epidural hematomas: a review of the literature and a comparison with operative cases. Acta Neurochir (Wien). 2004;146(2):103-10.
10. Groen RJ, Ponssen H. The spontaneous spinal epidural hematoma. A study of the etiology. J Neurol Sci. 1990;98(2-3):121-38.

11. Nagata K, Ariyoshi M, Ishibashi K, Hashimoto S, Inoue A. Chronic lumbar epidural hematoma in a patient who had spondylolysis at the third lumbar vertebra. Report of a rare case involving a seventeen-year-old adolescent. J Bone Joint Surg Am. 1998;80(10):1515-20.

12. Sang UH, Wilson CB. Postoperative epidural hematoma as a complication of anterior cervical discectomy. Report of three cases. J Neurosurg. 1978;49(2):288-91.

13. Tarlov IM. Spinal cord compression studies. III. Time limits for recovery after gradual compression in dogs. AMA Arch Neurol Psychiatry. 1954;71(5):588-97.

14. Tarlov IM, Herz E. Spinal cord compression studies. IV. Outlook with complete paralysis in man. AMA Arch Neurol Psychiatry. 1954;72(1):43-59.

15. Tarlov IM, Klinger H. Spinal cord compression studies. II. Time limits for recovery after acute compression in dogs. AMA Arch Neurol Psychiatry. 1954;71(3):271-90.

16. Vandermeulen EP, Van Aken $H$, Vermylen J. Anticoagulants and spinal-epidural anesthesia. Anesth Analg. 1994;79(6):1165-77.

17. Cabana F, Pointillart V, Vital J, Sénégas J. [Postoperative compressive spinal epidural hematomas. 15 cases and a review of the literature]. Rev Chir Orthop Reparatrice Appar Mot. 2000;86(4):335-45. 